\title{
CNTN-1 promotes docetaxel resistance and epithelial- to-mesenchymal transition via the PI3K/Akt signaling pathway in prostate cancer
}

Binshen Chen, Yiming Zhang, Chaoming Li, Peng Xu, Yubo Gao, Yawen Xu

Department of Urology, Zhujiang Hospital, Southern Medical University, Guangzhou, China

Submitted: 23 December 2018

Accepted: 5 March 2019

Arch Med Sci 2021; 17 (1): 152-165

DOI: https://doi.org/10.5114/aoms.2020.92939

Copyright $\odot 2020$ Termedia \& Banach

\section{Abstract}

Introduction: Therapy options for prostate cancer (PCa) typically are centered on docetaxel-based chemotherapy but are limited by the effects of multi-drug resistance. Recent advances have illustrated a role of contactin-1 (CNTN-1) in tumor chemoresistance, while the function and mechanism of CNTN-1 in the resistance of docetaxel in prostate cancer have not yet been elucidated.

Material and methods: Docetaxel (Dox)-resistant PCa cell lines of PC3 (PC3DR) and DU145 (DU145-DR) were established, and short hairpin RNA (shRNA) constructs targeting CNTN-1 were generated to analyze the effect of knockdown of CNTN-1 on PCa progression. Cell Counting Kit-8 (CCK-8), flow cytometry, wound-healing, transwell and western blotting analysis were used to analyze cell proliferation, apoptosis, migration, invasion and related protein expression levels, respectively.

Results: Knockdown of CNTN-1 in PC3-DR and DU145-DR cells attenuated cell proliferation, migration, invasion, EMT phenotype, and drug resistance, and increased cell apoptosis further reduced the tumorigenic phenotype. Knockdown of CNTN-1 resulted in an anti-tumor effect in the xenograft tumor model, and decreased activity of the phosphoinositide 3-kinase (PI3K)/ Akt signaling pathway both in vitro and in vivo.

Conclusions: The results of the present study suggest that downregulation of CNTN-1 may be an important mechanism to reverse chemoresistance in Dox-resistant PCa progression, thus shedding light on the development of novel anti-tumor therapeutics for the treatment of PCa.

Key words: contactin-1, epithelial-mesenchymal transition, docetaxel, chemoresistance, PI3K/Akt, prostate cancer.

\section{Introduction}

Prostate cancer ( $\mathrm{PCa}$ ) is considered as a common malignancy and one of the leading cancer-associated mortalities in men. In the United States, it is estimated that more than 220,000 newly diagnosed cases and approximately 27,000 deaths occur annually [1]. Worse still, in spite of most patients with metastatic PCa responding to androgen deprivation therapy, all of them unavoidably gradually transform into incurable castration-resistant prostate cancer (CRPC), which is defined as the fatal form of PCa [2-4]. As one of the taxol-derived drugs, docetaxel (Dox)

\author{
Corresponding author: \\ Yawen Xu \\ Department of Urology \\ Zhujiang Hospital \\ Southern Medical \\ University \\ 253 Gongye \\ Middle Avenue \\ 510282, Guangzhou \\ Guangdong Province \\ China \\ E-mail: yawenxu72@163.com
}


has been extensively used as standard first-line chemotherapy in CRPC patients for more than a decade $[5,6]$. However, Dox merely lengthens the moderate survival as patients inevitably obtain drug resistance and ultimately die of this disease [7-10]. Therefore, it is worth identifying novel promising therapeutic targets and clarifying underlying molecular mechanisms of Dox resistance to PCa.

As a dynamically reversible conversion cellular process, epithelial-mesenchymal transition (EMT) promotes the transformation of epithelial cells into mesenchymal cells in which mesenchymal cells acquire cell-cell adhesion, invasiveness and metastasis of the malignancy, thereby resulting in cancer cell mobility, anti-apoptosis abilities, and even poor prognosis [11-14]. EMT is central in embryogenesis, chronic inflammation, tissue remodeling, tumor metastasis, and multiple fibrotic diseases [15]. Recent studies implied that EMT is not a necessary prerequisite for the metastatic process but is an essential chemical resistance development process in multiple cancer cells [16-18]. In addition, EMT was found to participate in the formation of tumor stem cell phenotype in some drug-resistant cancer cells [12, 19-21]. Upstream transcription factors such as Snail, Slug, Twist and ZEB1 regulate cancer cells through different pathways to obtain increase of mesenchymal cell markers (N-cadherin, vimentin and fibronectin) and deletion of epithelial cell markers (E-cadherin), which ultimately lead to the EMT phenotype of drug-resistant cancer cells [16]. Moreover, EMT has also been shown to be involved in the PI3K/ Akt signaling pathway [22, 23], which is also involved in Dox resistance in PCa $[20,24,25]$. As a consequence, new measures to restrain EMT or promote mesenchymalepithelial transition (MET) are essential to enhance the therapeutic effect of PCa. As one of the neuronal cell adhesion molecules, contactin-1 (CNTN-1), which is composed of N-CAM, L1 and Nr-CAM, belongs to the immunoglobulin superfamily [26], and has been identified as a tumor oncogene, which is found to be up-regulated and involved in several cancers including gastric [27], lung $[28,29]$ and prostate cancer [30,31]. Moreover, emerging evidence has indicated that CNTN-1 is associated with cisplatin chemo-resistance in lung cancer [29], suggesting that CNTN-1 may serve as a potential drug resistance-associated tumor gene. Additionally, whether CNTN-1 is related to Dox resistance in PCa is still not fully elucidated.

The current study intended to investigate the role of CNTN-1 in docetaxel resistance in PCa. Dox-resistant PCa cell lines (PC3-DR and DU145-
DR) and xenograft mouse models were respectively established for in vitro and in vivo experiments. The results illustrated that CNTN-1 could regulate EMT phenotype and malignant progression of Dox-resistant PCa cells by means of activating PI3K/Akt signaling. Regulation of expression of CNTN-1 might provide effective therapeutic treatment for drug resistance and malignant progress in Dox-resistant PCa.

\section{Material and methods}

\section{Cell culture and reagents}

Human prostate cell lines PC3 and DU145 were originally obtained from American Type Culture Collection (ATCC) (Manassas, VA, USA). Cells were maintained in RPMI 1640 medium (Gibco, Rockville, MD, USA) supplemented with $10 \%$ fetal bovine serum (Sigma Aldrich, Oakville, ON) and $100 \mathrm{U} / \mathrm{ml}$ of penicillin/streptomycin (Life Technologies, Burlington, $\mathrm{ON}$ ) at $37^{\circ} \mathrm{C}$ in a humidified atmosphere with $5 \% \mathrm{CO}_{2}$. Docetaxel was bought from Sigma-Aldrich (Sigma-Aldrich, St. Louis, MO).

\section{Establishment of Dox-resistant cells}

PC3 and DU145 Dox-resistant cell lines were selected based on constant exposure of the parental cells to docetaxel in stepwise dose incremental concentrations of docetaxel from $5 \mathrm{nM}$ to $200 \mathrm{nM}$ as previously described [32]. After successful establishment, these resistant cell lines were continuously cultivated in media that contained docetaxel and were respectively named as "PC3-DR" and "DU145-DR".

\section{Cell transfection}

PC3-DR and DU145-DR cells were transfected with shRNA control (shNC, sequences for shNC: 5'-TTCTCCGAACGTGTCACGTAA-3') or shRNA targeting CNTN-1 (shCNTN-1, sequences for shCNTN-1: 5'-CCCGGTTTACAAATGGAGAAT-3'). The recombinant lentivirus with $\mathrm{CNTN}$-1-gene was produced by co-transfection of 293T cells with helper plasmids (psPAX2 and pMD2G) with Lipofiter (Hanbio, China) as previously described [27].

\section{Cell viability assay}

Cell viability was assessed using the CCK-8 proliferation detection kit (Dojindo, Tokyo, Japan). Briefly, a total of approximately $5 \times 10^{4}$ cells were seeded in 96-well plates; $24 \mathrm{~h}$ after incubation, Dox at different concentrations (5-200 nM) was added to the culture medium for another $48 \mathrm{~h}$. Then $10 \mu \mathrm{l}$ of CCK- 8 solution was added to each well and cultivated for $4 \mathrm{~h}$. The absorbance of the reaction system was measured spectrophotometrically at $450 \mathrm{~nm}$. 


\section{Cell apoptosis analysis}

Flow cytometry analysis for apoptosis was performed with Annexin V FITC-propidium iodide (PI) staining assay (Invitrogen, California, USA) based on the manufacturer's instructions.

\section{Wound-healing assay}

$5 \times 10^{5}$ cells were seeded into a 6 -well plate and cultivated until reaching about $90 \%$ confluence. A straight wound line was made using a sterile $200 \mu$ l pipette tip. Cells with different treatments were cultured in the medium for an additional $24 \mathrm{~h}$, and then wound recovery was evaluated under a light microscope (Olympus BX61, Japan).

\section{Cell invasion assay}

$3 \times 10^{4}$ cells were seeded in the upper chamber pre-coated with Matrigel (BD, Franklin Lakes, NJ, USA) and incubated with serum-free medium. The lower chamber was filled with medium supplemented with $10 \%$ FBS. After $48 \mathrm{~h}$ of incubation, the invasive cells which passed through the Matrigel membrane in the lower chamber were fixed with $4 \%$ paraformaldehyde and stained with crystal violet (Cat \# C-3886, Sigma-Aldrich, St. Louis, $\mathrm{MO})$, then photographed under a light microscope (Olympus BX61, Japan) at a magnification of 100x in five random fields across the center and the periphery of the membrane.

\section{Western blotting analysis}

Cells were lysed with lysis buffer containing protease inhibitors $(50 \mathrm{mM}$ Tris- $\mathrm{HCl} \mathrm{pH} 8,50 \mathrm{mM}$ $\mathrm{NaCl}, 0.5 \% \mathrm{NP}-40$ ). Equal amounts of protein were separated by sodium dodecyl sulfate-polyacrylamide gel electrophoresis (SDS-PAGE) gels, and transferred to PVDF membranes. After blocking with $5 \%$ nonfat milk, the membranes were then treated with rabbit polyclonal anti-CNTN-1 (1: 500, Proteintech), anti-Akt (1: 1000, Cell signaling Technology), anti-phospho-Akt antibody (1 : 1000; Cell signaling Technology), anti-E-cadherin 1 : 1000; Cell signaling Technology), anti$\mathrm{N}$-cadherin (1: 1000; Cell signaling Technology), anti-vimentin (1 : 1000; Cell signaling Technology), anti-fibronectin (1:1000; Santa Cruz Biotechnology), anti-CD44 (1 : 2000; Abcam), anti-OCT4 (1 : 2000; Abcam), anti-Nanog (1 : 2000; Abcam), anti- $\beta$-actin ( $1: 500 ;$ Abcam) overnight at $4^{\circ} \mathrm{C}$. Following washing, the membranes were exposed to HRP-conjugated goat anti-rabbit (1 : 2000, Abcam) secondary antibodies and target protein bands were detected with the ECL detection system.

\section{In vivo study}

All studies involving animals were conducted according to the protocol approved by the Institu- tional Animal Care and Use Committee of Southern Medical University (Guangzhou, China). Forty six-week old female nude mice were purchased from the animal house of the Faculty of Medicine, Southern Medical University (Guangzhou, China). $100 \mu \mathrm{l}$ of PC3-DR cell suspension $\left(5 \times 10^{6} / 100 \mu \mathrm{l}\right)$ which was transfected with shNC or shCNTN-1 was subcutaneously injected into the right flank of mice. At 7 days after inoculation, mice $(n=10$ per group) were treated with a dose of $10 \mathrm{mg} / \mathrm{kg}$ docetaxel or equivalent PBS solution containing 0.5\% DMSO via intraperitoneal injection every 3 days. Tumor volumes were examined every 3 days using the formula: volume $=0.5 \times$ length $\times$ width ${ }^{2}$. At 28 days after the subcutaneous injection, mice were sacrificed and tumors were isolated and weighed.

\section{Ethics}

The present study was approved by the Ethical Committee of Southern Medical University (Guangzhou, China).

\section{Statistical analysis}

All quantitative data were presented as mean \pm SD and analyzed using SPSS 16.0 software (SPSS Inc., Chicago, IL, USA). The comparison of significances was evaluated by a two-sided Student's $t$ test for two groups and one-way ANOVA test for three or more groups. All experiments were repeated at least three times. $P<0.05$ was considered as statistically significant.

\section{Results}

\section{Dox-resistant prostate cancer cells exhibited up-regulated CNTN-1 expression and an EMT phenotype}

Aiming to identify the function of CNTN-1 in Dox-resistant prostate cancer, PC3-DR and DU145-DR Dox-resistant cell lines of prostate cancer were established. As shown in Figures 1 A-C, the PC3-DR and DU145-DR cell lines showed obviously more powerful resistance to docetaxel and a lower apoptosis rate than the parental cells, suggesting that Dox-resistant PCa cell lines were induced successfully. In addition, the CNTN-1 protein level in the PCa cells was detected by western blotting. The results demonstrated that both PC3DR and DU145-DR displayed increased protein expression of CNTN-1 compared with the parental cells (Figure $1 \mathrm{D}$ ). These data described above suggested that CNTN-1 may play a critical role in docetaxel resistance and chemo-resistance of PCa. To supply direct evidence that EMT development is correlated with Dox resistance, the cellular morphological changes were observed. Moreover, 
A

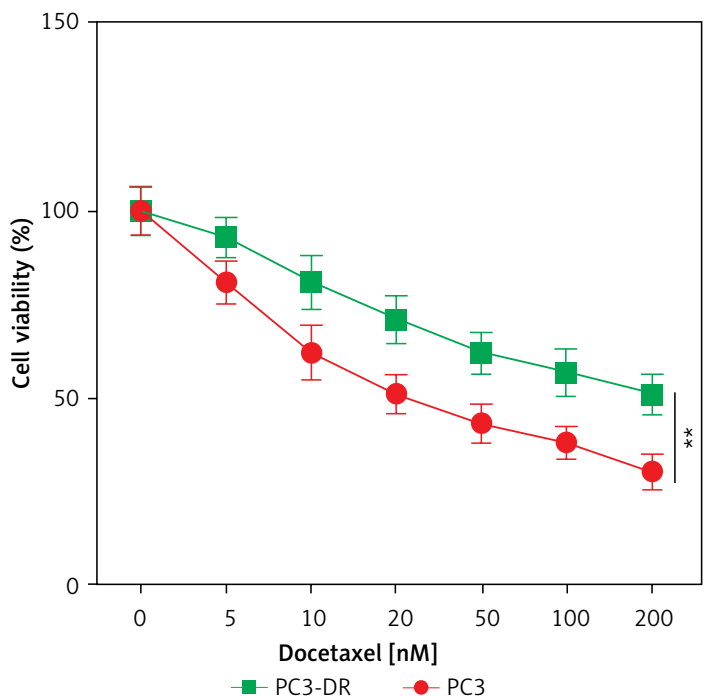

B

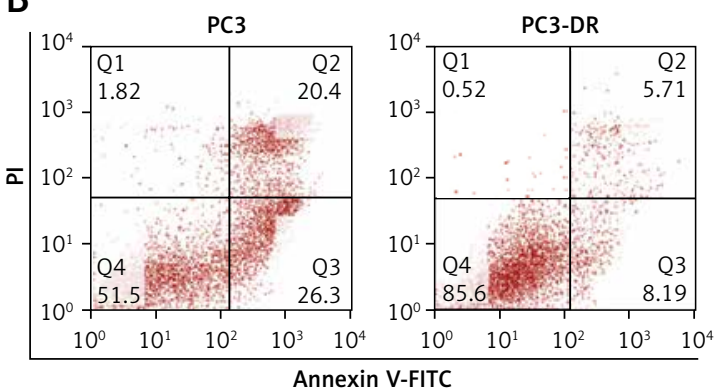

C

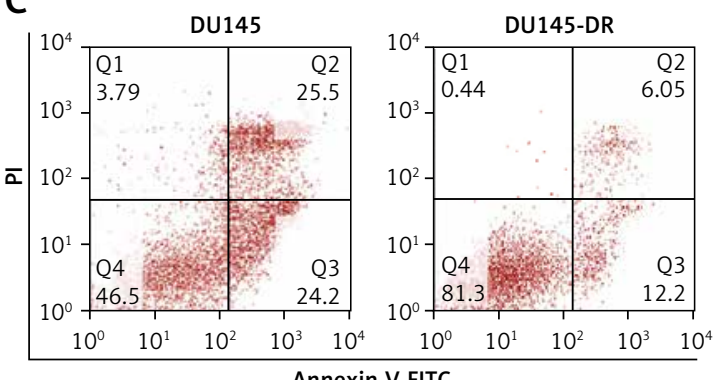

D PC3 PC3-DR DU145 DU145-DR

CNTN-1

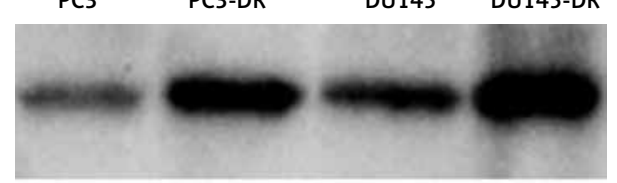

$\beta$-actin

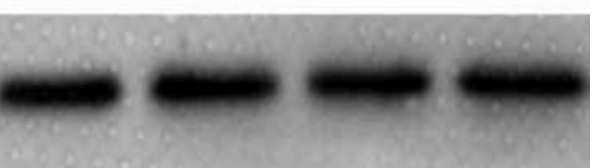

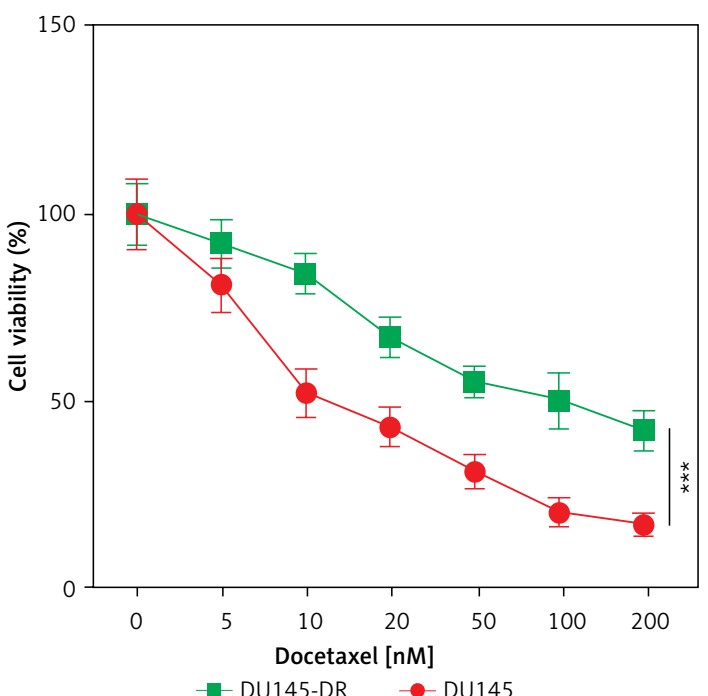

- DU145-DR - DU145
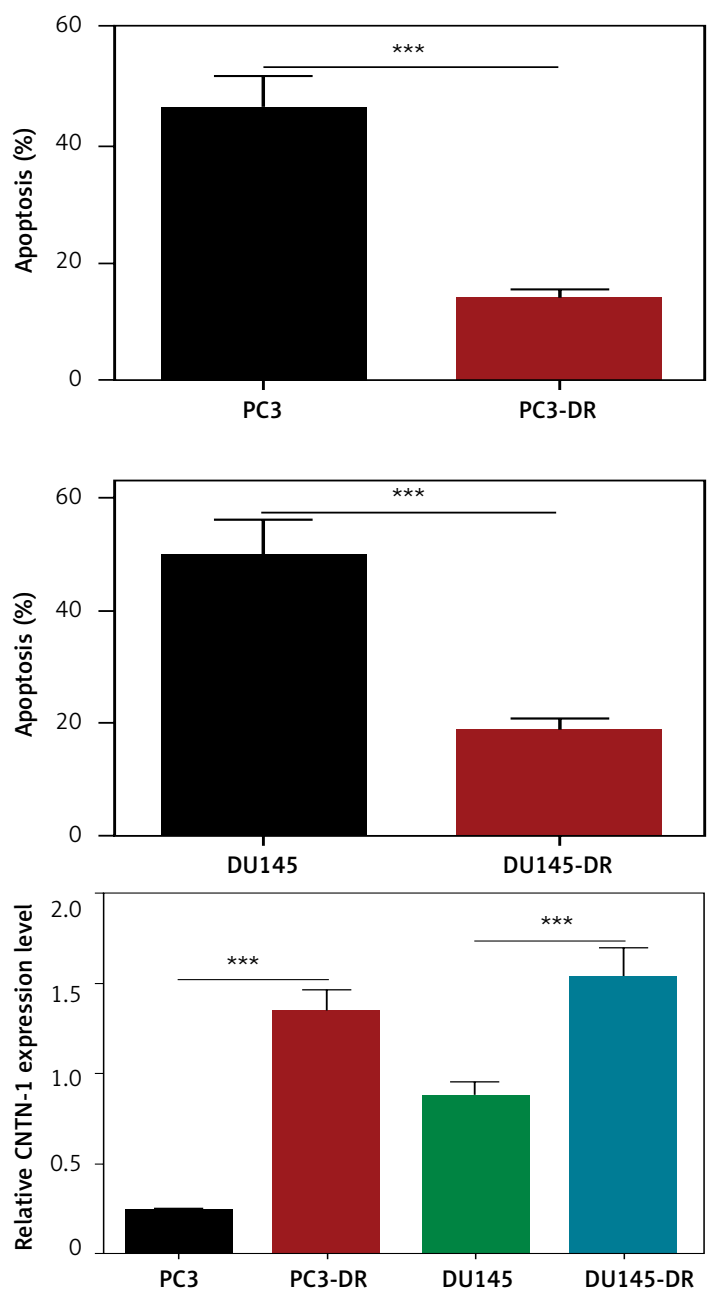

Figure 1. Dox-resistant prostate cancer cells exhibited up-regulated CNTN-1 expression and an EMT phenotype. A - Cells were treated with different concentrations of docetaxel (0-200 nM) for $48 \mathrm{~h}$ and the cell viability was analyzed by CCK-8 assay. B, C - Cell apoptosis was evaluated by flow cytometry. D - The protein level of CNTN-1 was determined by western blotting

Data are presented as the mean $\pm S D .{ }^{*} P<0.05,{ }^{* *} p<0.01,{ }^{* * *} p<0.001$. 
$E$

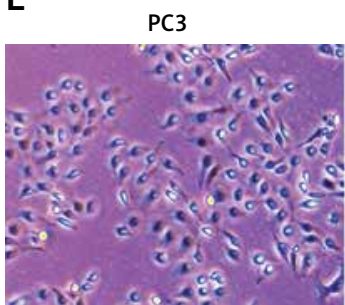

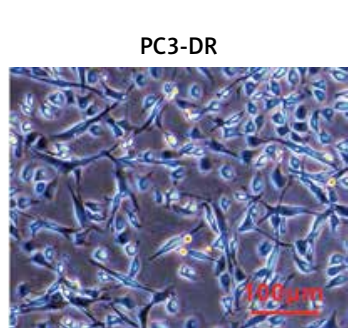

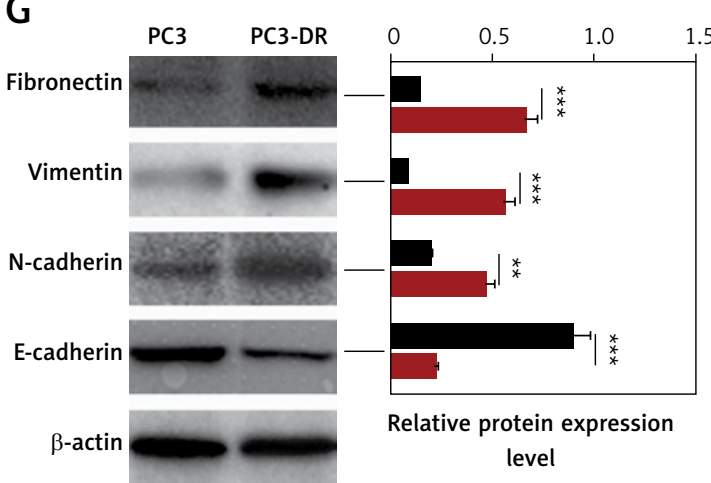

$\mathrm{F}$
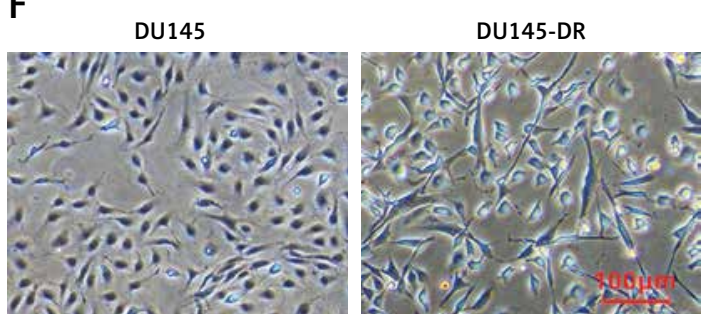

\section{$\mathrm{H}$}

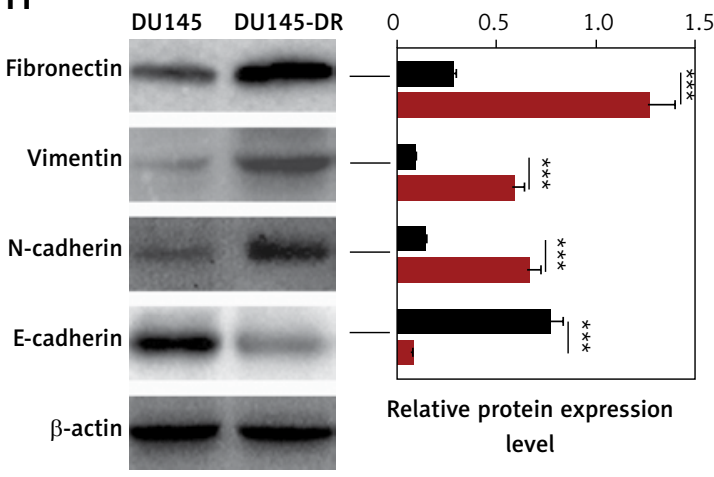

Figure 1. Cont. E - The cell morphology was evaluated by microscopic observations (magnification 200x, bar = $100 \mu \mathrm{m}) . \mathrm{F}$ - The protein expression levels of EMT-related markers (fibronectin, vimentin, $\mathrm{N}$-cadherin and E-cadherin) were evaluated by western blotting

Data are presented as the mean $\pm S D .{ }^{*} P<0.05,{ }^{* *} p<0.01,{ }^{* *} p<0.001$.

A
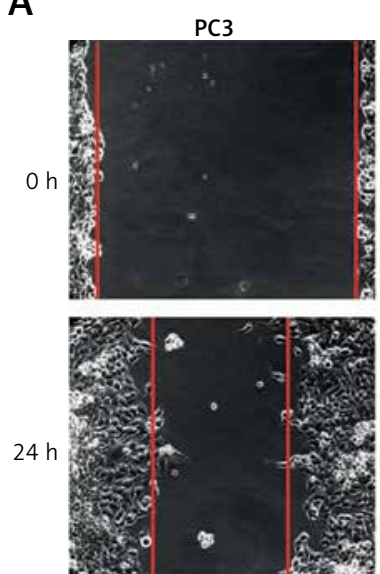

B

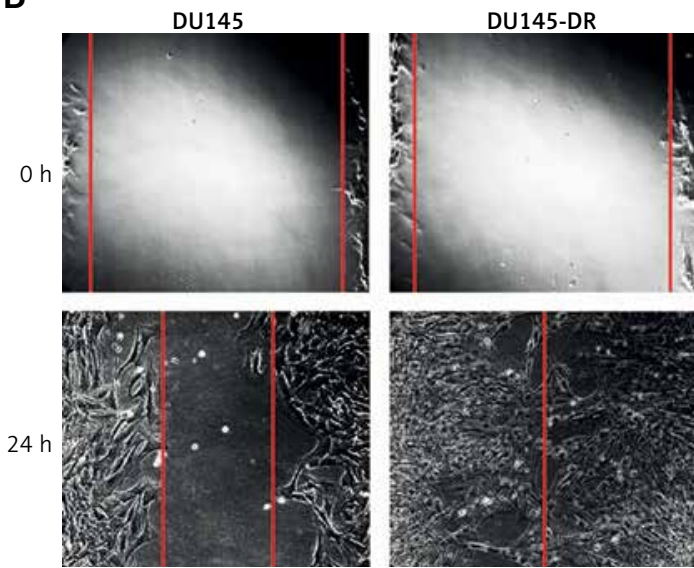

PC3-DR

DU145-DR
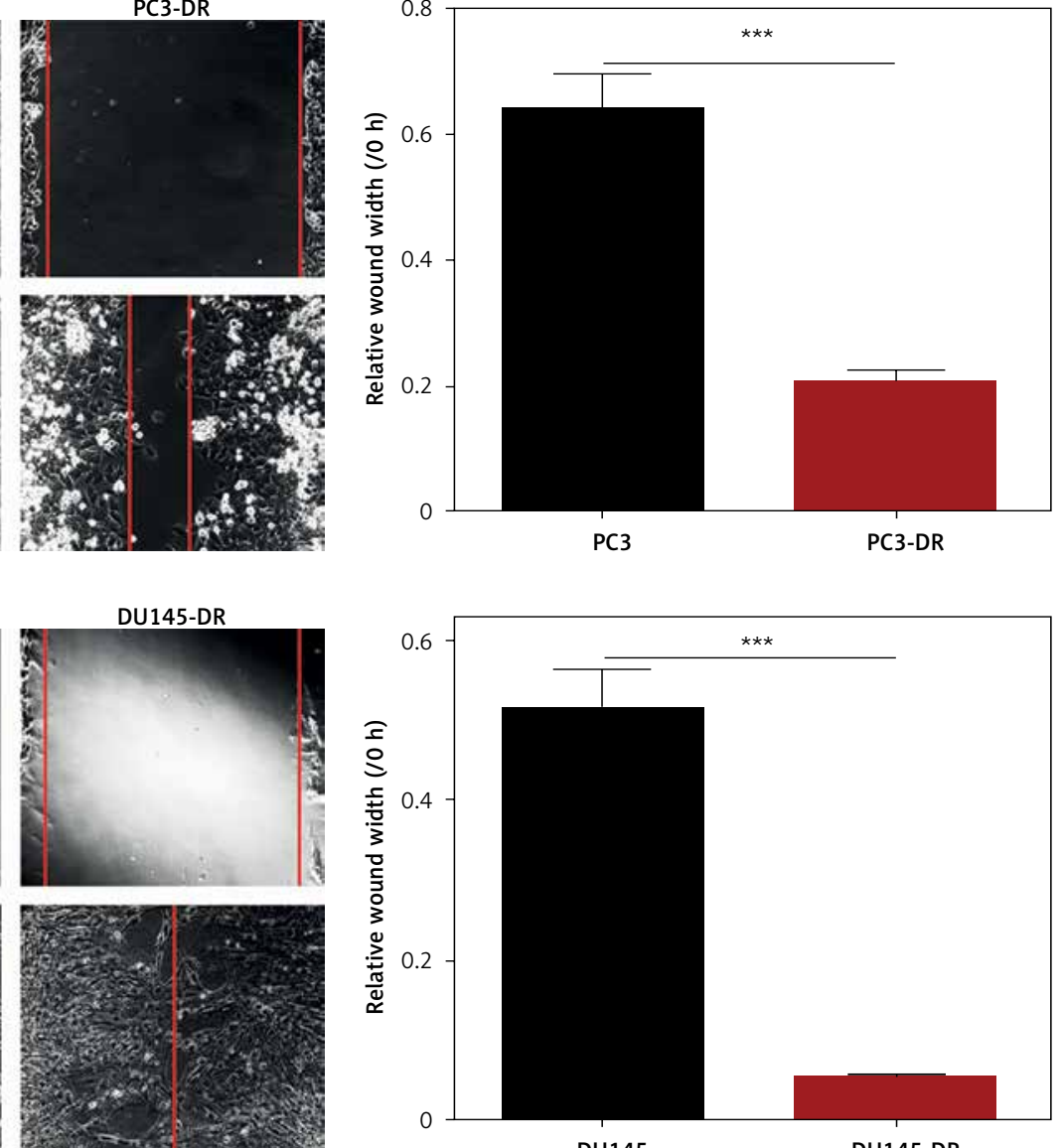

Figure 2. Dox-resistant prostate cancer cells exhibited cellular malignant phenotype. A, B - Cell migration was evaluated by wound-healing assay

Data are presented as the mean $\pm S D .{ }^{*} P<0.05,{ }^{* *} p<0.01,{ }^{* * *} p<0.001$. 
C
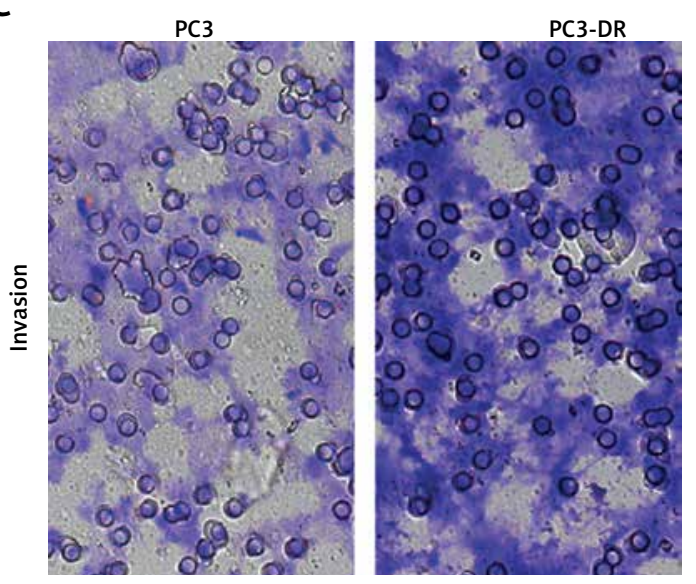

D
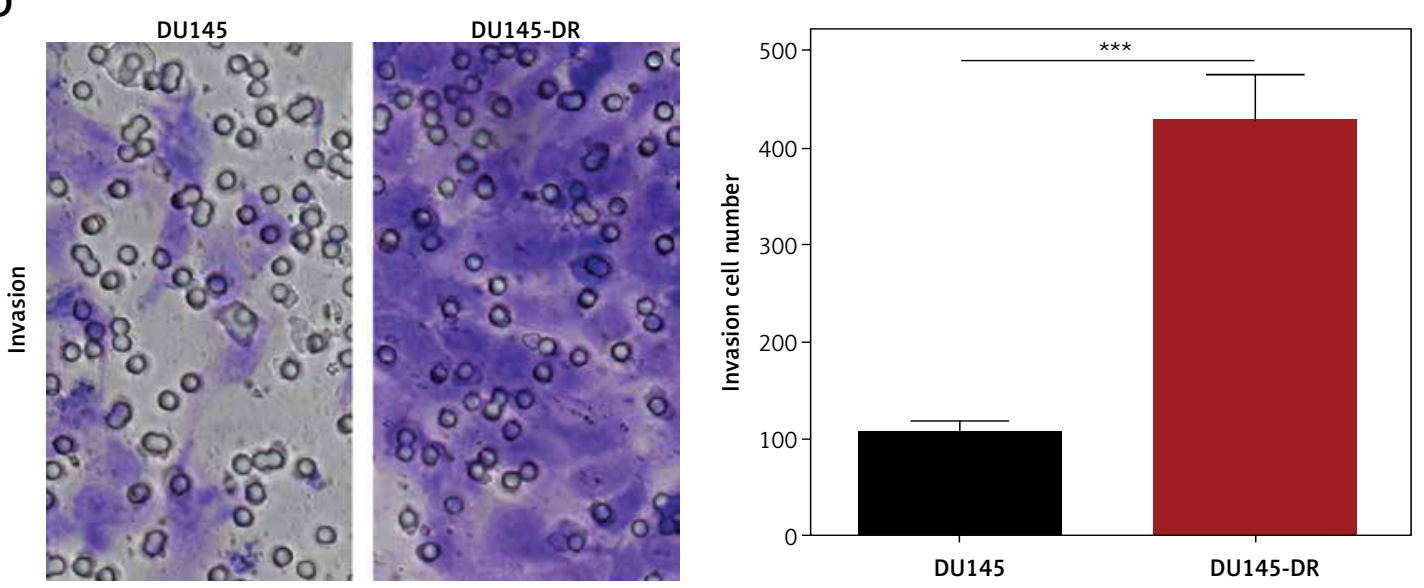

$E$
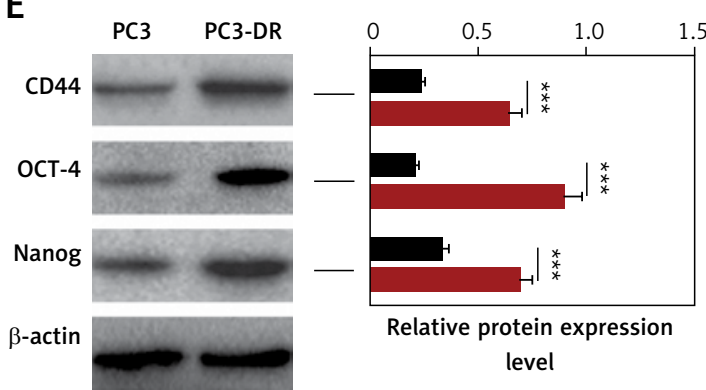

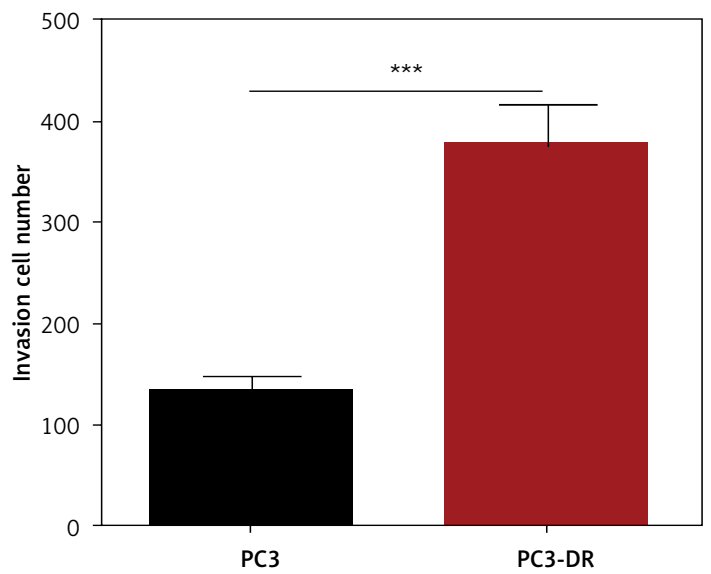

PC3

$\mathrm{F}$

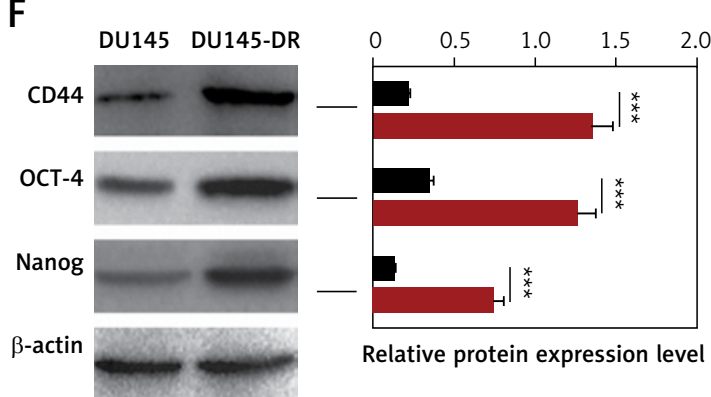

Figure 2. Cont. C - Cell invasion was examined by transwell assay. D - Cell invasion was examined by transwell assay. E, F - Protein levels of CD44, OCT-4 and Nanog were detected by western blotting

Data are presented as the mean $\pm S D$. ${ }^{*} P<0.05,{ }^{* *} p<0.01,{ }^{* * *} p<0.001$

the protein levels of fibronectin, vimentin, N-cadherin and E-cadherin also were detected. The resistant cells sustained Dox resistance when incubated for a period of time in a medium without docetaxel and displayed a slender and irregular separate fibroblastic morphology in comparison with the parental cells (Figures $1 \mathrm{E}-\mathrm{F}$ ). Consistent with morphological characteristics, the resistant cells also showed increased protein expression of fibronectin, vimentin and $\mathrm{N}$-cadherin but protein expression of E-cadherin was comparable with the parental cells (Figures $1 \mathrm{G}-\mathrm{H}$ ). Collectively, these data described above demonstrate that PC3-DR and DU145-DR cells obtain an EMT phenotype.

\section{Dox-resistant prostate cancer cells exhibited cellular malignant phenotype}

EMT activation can lead to the malignant phenotype of the tumor, including invasion, metastasis, stem cell characteristics, and resistance to apoptosis [12, 33-35]. Hence, the malignant progression of Dox-resistant and parental PCa cells was detected, respectively. The results of 
wound-healing and transwell migration/invasion indicated that PC3-DR and DU145-DR cell lines show more significant migration and invasion abilities compared to the parental PCa cells (Figures $2 \mathrm{~A}-\mathrm{D}$ ). Moreover, the protein levels of pluripotent markers including CD44, OCT-4 and Nanog were increased in PC3-DR and DU145-DR cell lines compared to the parental cells (Figures $2 \mathrm{E}-\mathrm{F}$ ). These observations demonstrated that EMT is

A
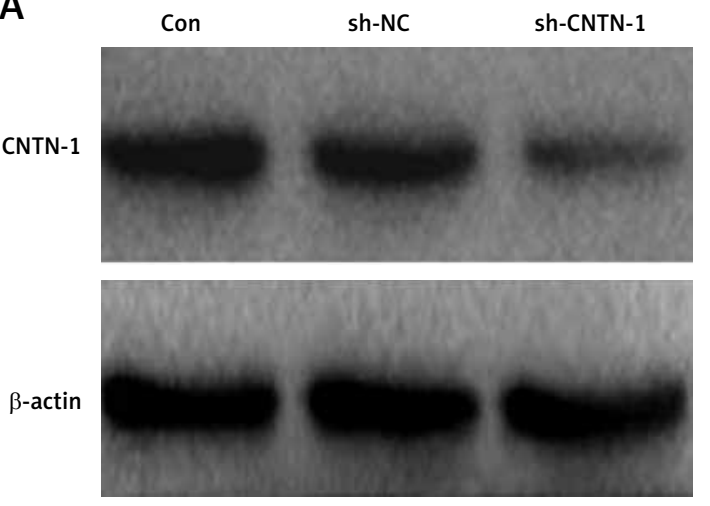

PC3-DR
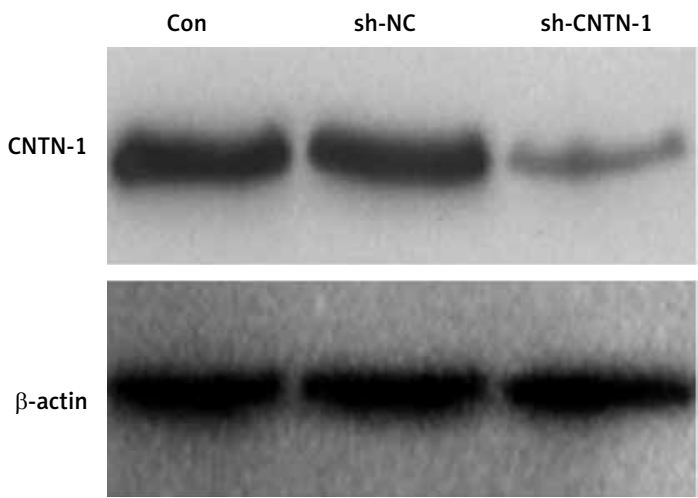

DU145-DR

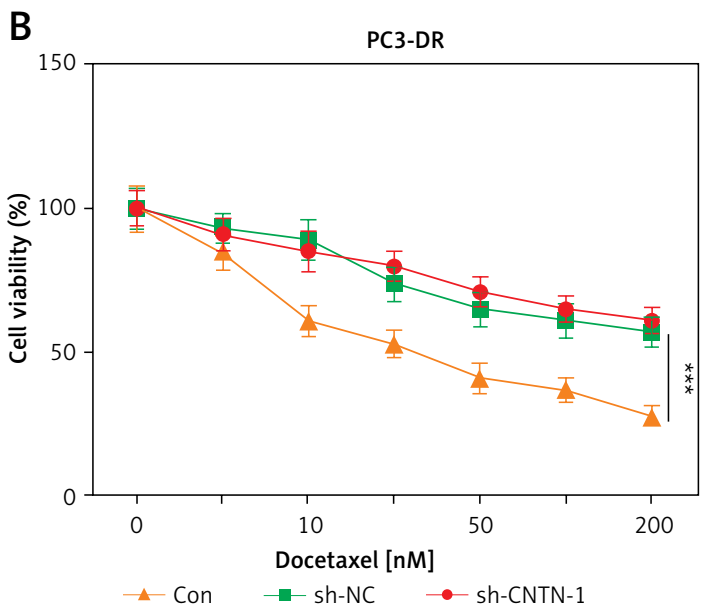

involved in Dox resistance and aggravates tumor malignant biological behaviors in PCa cells.

\section{Silencing CNTN-1 inhibited proliferation and EMT of Dox-resistant prostate cancer cells}

On account of the results described above, we suspected that the proliferation and EMT pheno-
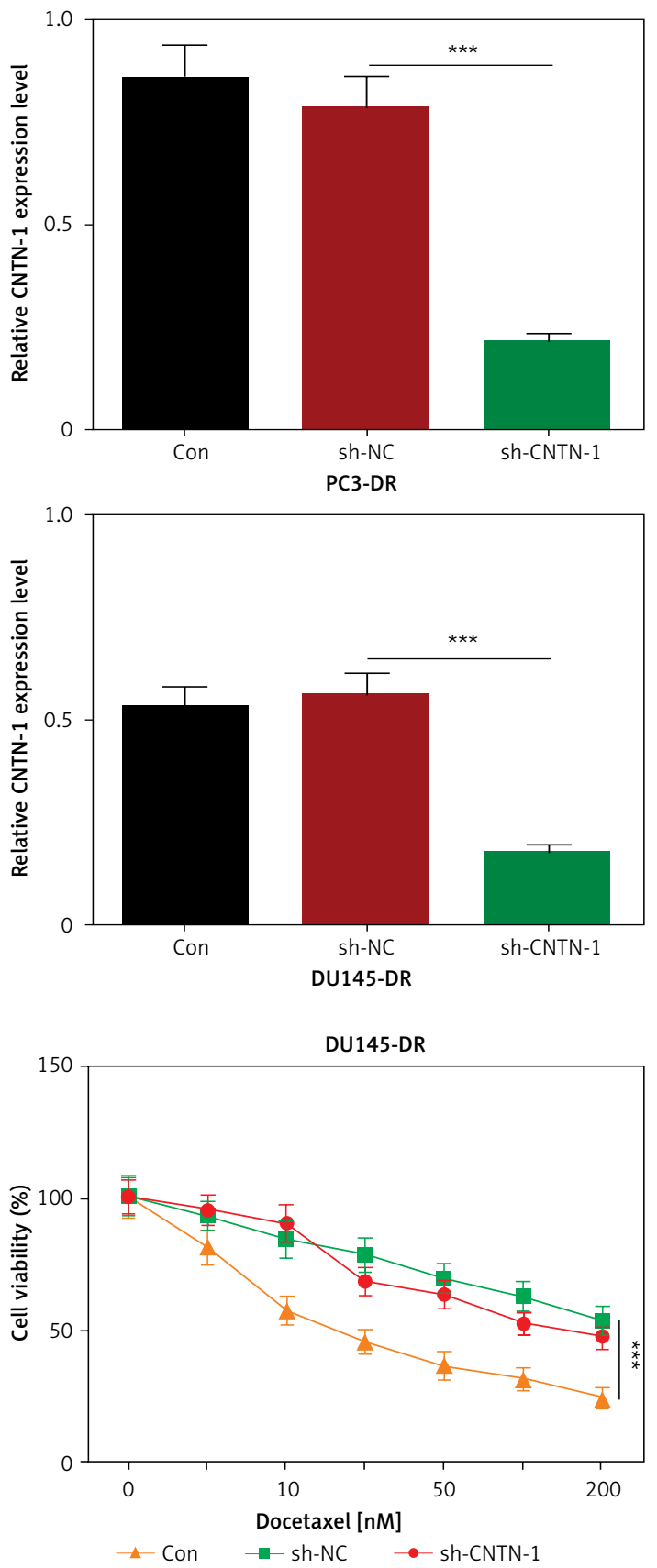

Figure 3. Silencing CNTN-1 inhibited proliferation and EMT of Dox-resistant prostate cancer cells. A - Transfection efficiency of CNTN-1 knockdown in PC3-DR and DU145-DR cells was analyzed by western blotting. B - PC3-DR and DU145-DR cells transfected by shCNTN-1 with different concentrations of docetaxel (0-200 nM) treatment and the cell viability was determined by CCK- 8 assay

Data are presented as the mean $\pm S D .{ }^{*} P<0.05,{ }^{* *} p<0.01,{ }^{* * *} p<0.001$. 
type may be interrelated with CNTN-1. To verify the hypothesis, CNTN-1 was successfully silenced in both PC3-DR and DU145-DR cells. The protein levels of CNTN-1 in both PC3-DR and DU145-DR cells were confirmed by western blotting (Figure 3 A). The CCK-8 assay revealed that silencing CNTN-1 in PC3-DR and DU145-DR cell lines visibly increased the sensitivity of Dox-resistant PCa cells to docetaxel compared with the shNC group
(Figure 3 B). Flow cytometry analysis further indicated that the shCNTN-1 group had an obviously elevated apoptosis rate in contrast to the shNC group in both PC3-DR and DU145-DR cell lines (Figure $3 \mathrm{C}$ ). Then, EMT-associated morphological changes were observed and the results showed that shCNTN-1 cells were characterized by a similar round epithelial phenotype in comparison with shNC cells, which displayed a slender and irregular
C

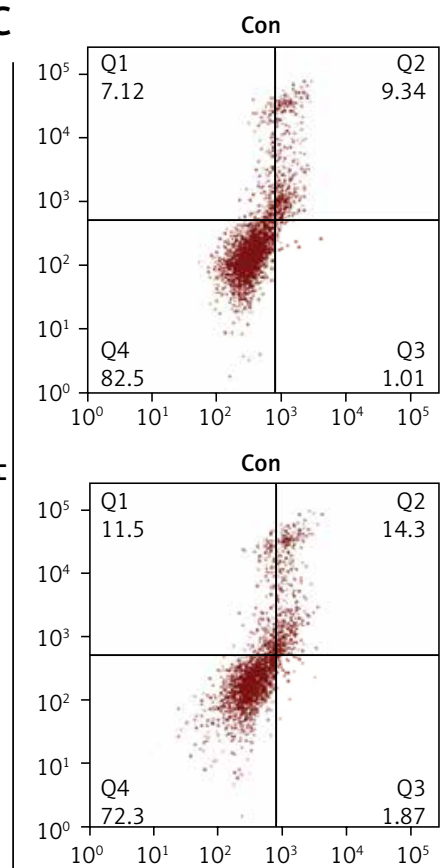

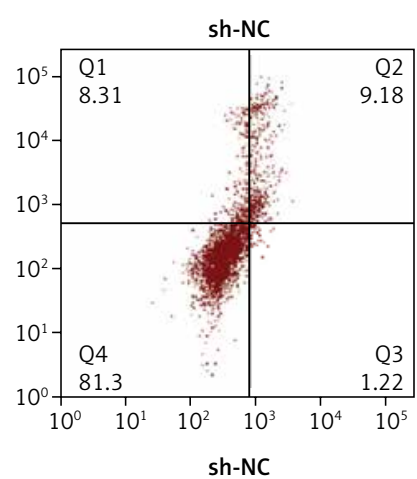

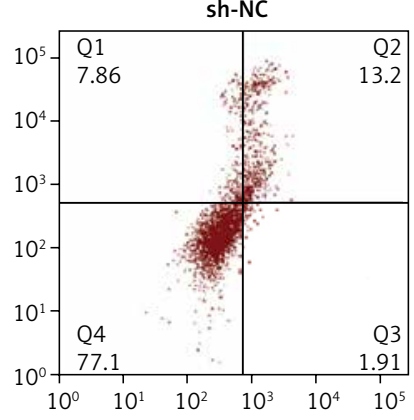

Annexin V-FITC
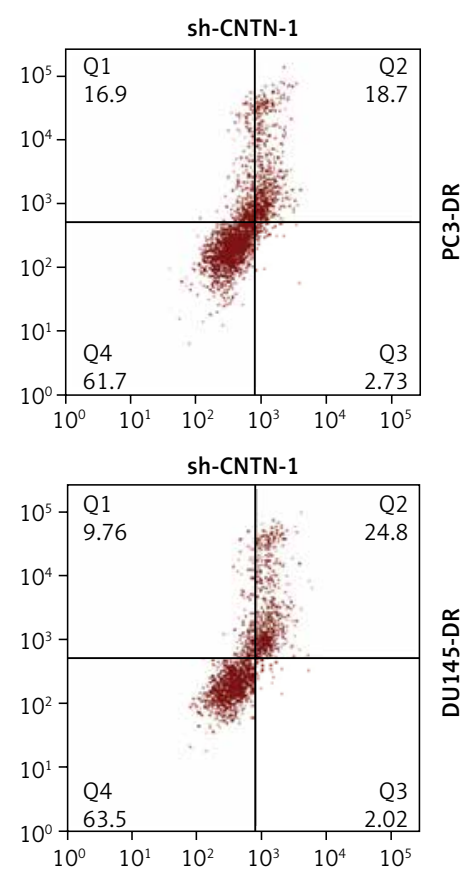
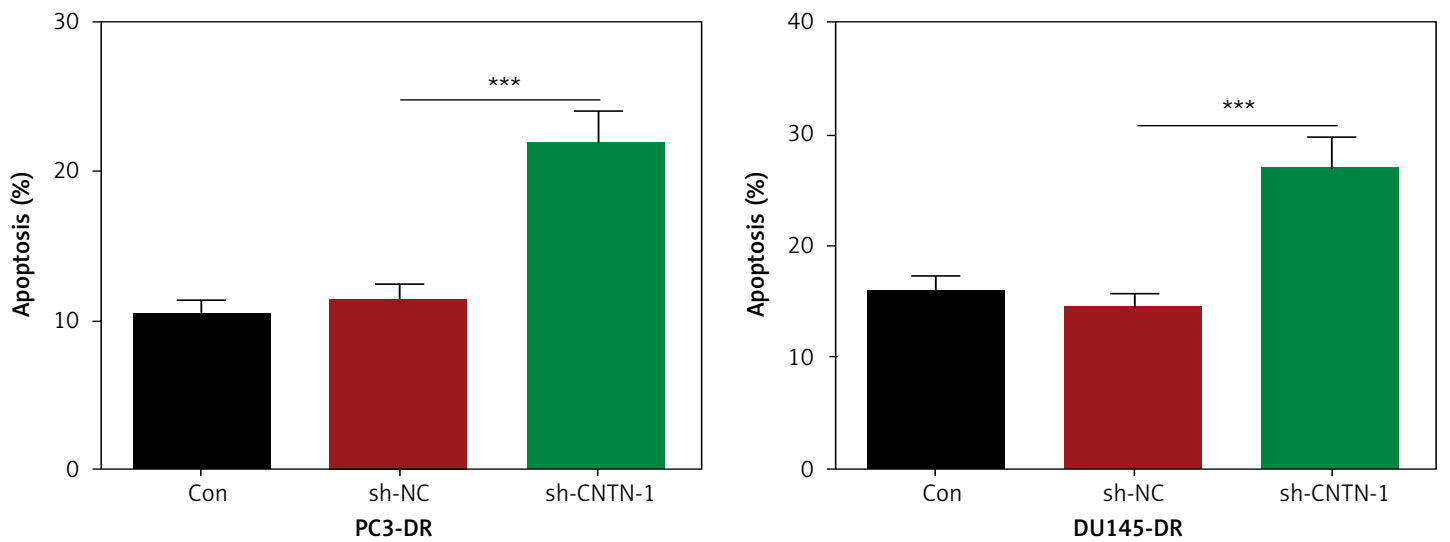

D
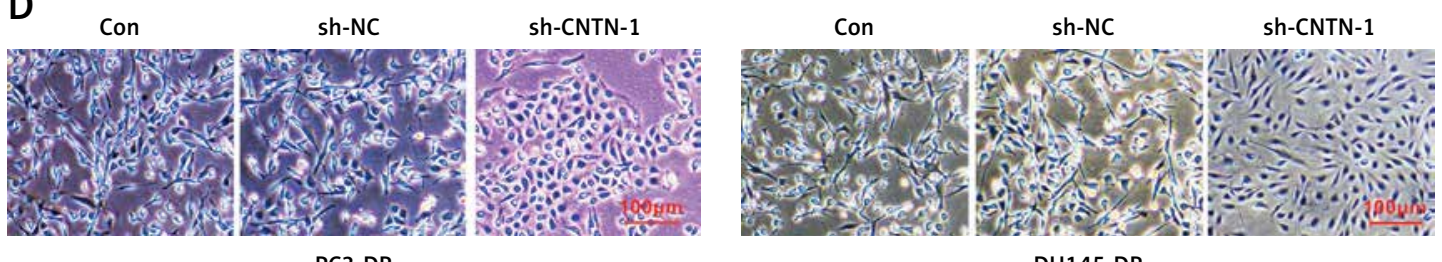

DU145-DR

Figure 3. Cont. C - Cell apoptosis was analyzed by flow cytometry. D - Cell morphology was evaluated by microscopic observations (magnification: 100x, bar $=200 \mu \mathrm{m}$ )

Data are presented as the mean $\pm S D .{ }^{*} P<0.05,{ }^{* *} p<0.01,{ }^{* * *} p<0.001$. 
mesenchymal cell morphology (Figure 3 D). Moreover, the protein levels of EMT-related markers were detected by western blotting. As revealed, CNTN-1 silencing significantly attenuated the expression level of mesenchymal markers including fi- bronectin, $\mathrm{N}$-cadherin and vimentin, but increased the epithelial marker E-cadherin (Figure $3 \mathrm{E}$ ). Therefore, it is conceivable that knockdown of CNTN-1 hindered proliferation and EMT in Dox-resistant PCa cells.

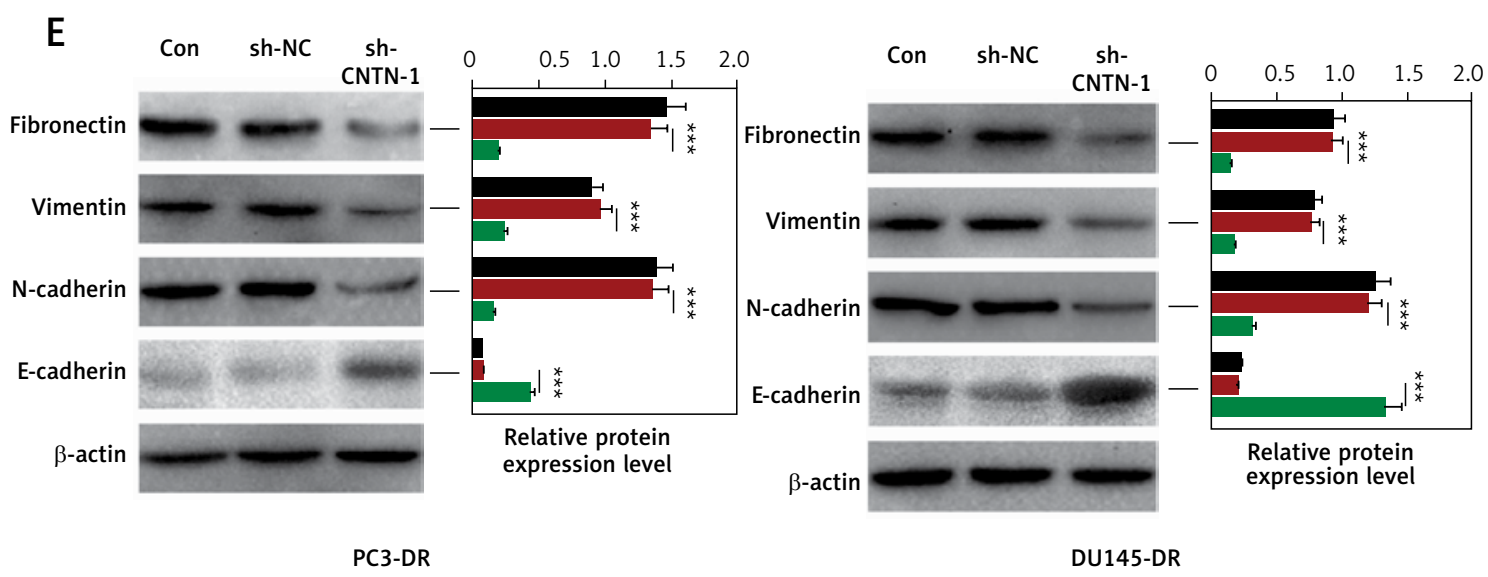

Figure 3. Cont. E - Protein levels of EMT-related markers (fibronectin, vimentin, N-cadherin and E-cadherin) were estimated using western blotting

Data are presented as the mean $\pm S D .{ }^{*} P<0.05,{ }^{* *} p<0.01,{ }^{* * *} p<0.001$.

A
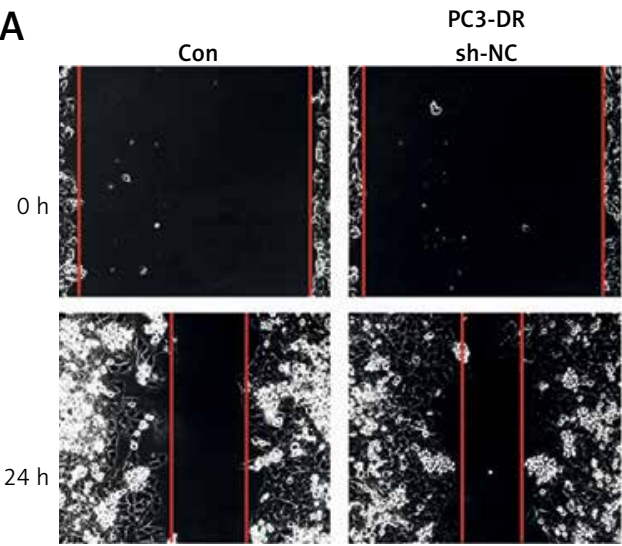

B
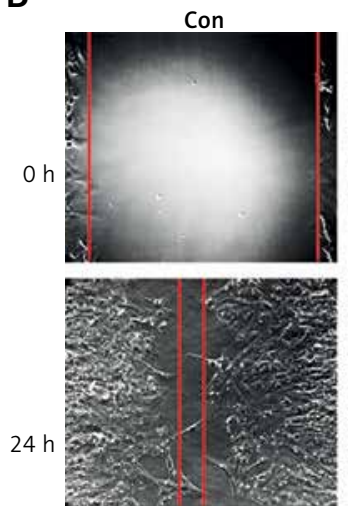

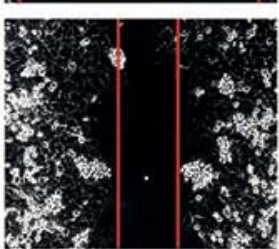

DU145-DR
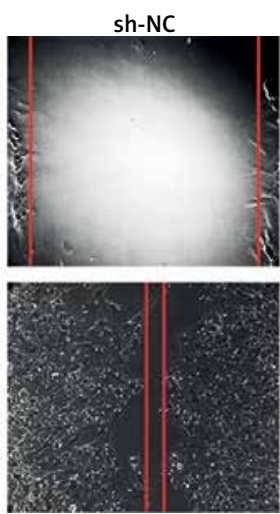

sh-CNTN-1

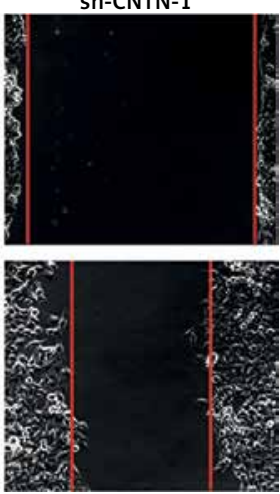

sh-CNTN-1

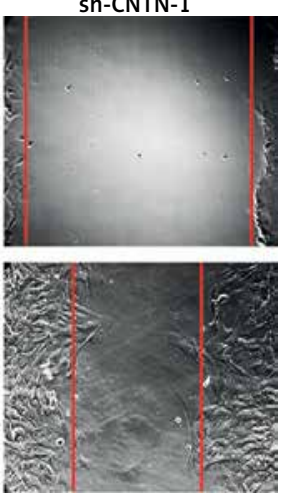

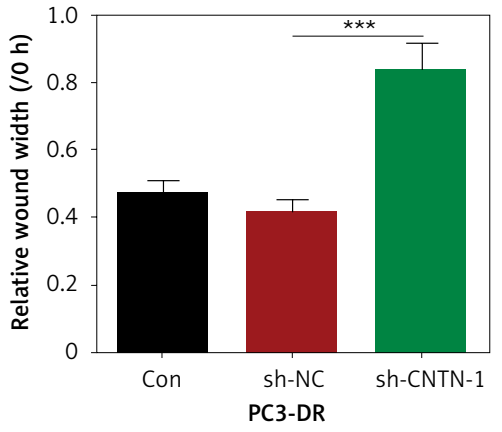

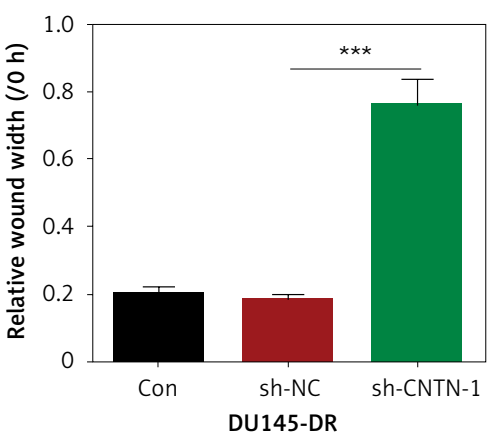

Figure 4. Silencing CNTN-1 inhibited cellular malignant phenotype of Dox-resistant prostate cancer cells. A, B - Cell migration was evaluated by wound-healing assay

Data are presented as the mean $\pm S D .{ }^{*} P<0.05,{ }^{* *} p<0.01,{ }^{* * *} p<0.001$. 
Silencing CNTN-1 inhibited cellular malignant phenotype of Dox-resistant prostate cancer cells

Given that CNTN-1 is involved in EMT phenotype, we next investigated whether CNTN-1 could affect the malignant phenotype of PC3-DR and DU145-DR cell lines. The ShCNTN-1 group showed an obvious reduction of migratory and invasive abilities compared to the shNC group both in PC3-DR and DU145-DR cell lines (Figures 4 A-D). In addition, the shCNTN-1 group also suppressed the protein expression level of pluripotent markers including CD44, OCT-4 and Nanog in contrast to shNC cells (Figures 4 E, F). Collectively, the results above suggested that knockdown of CNTN-1 reduced malignant behaviors in Dox-resistant PCa cells.

CNTN-1 regulates EMT and Dox resistance via mediating PI3K/Akt signaling

$\mathrm{PI}$ IK/Akt signaling is proved to be involved in cell proliferation, EMT and chemo-resistance in
C

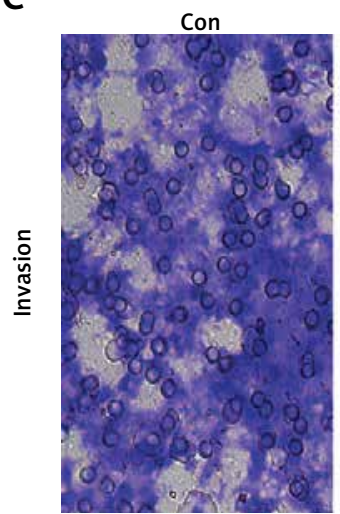

D

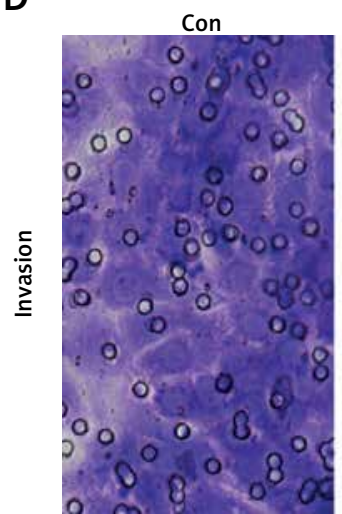

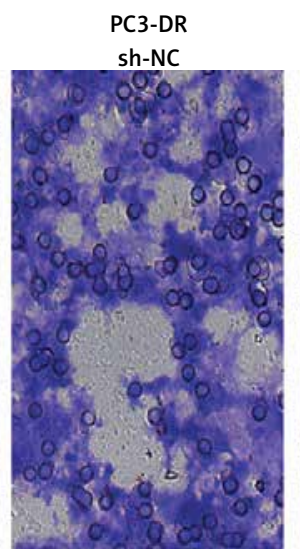

DU145-DR

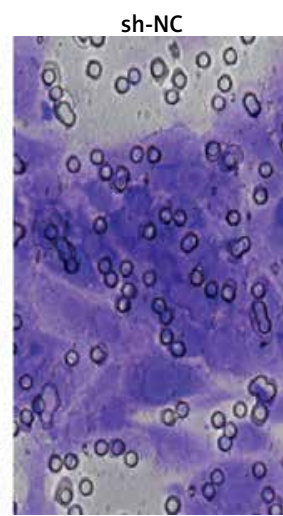

Sh-CNTN-1

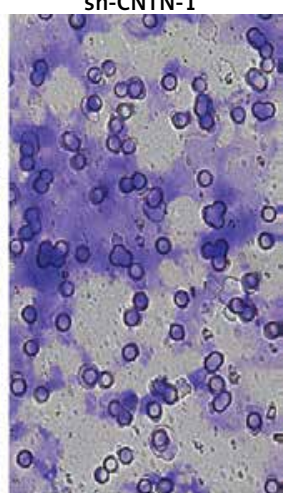

Sh-CNTN-1

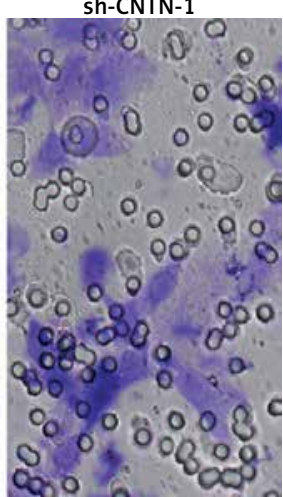

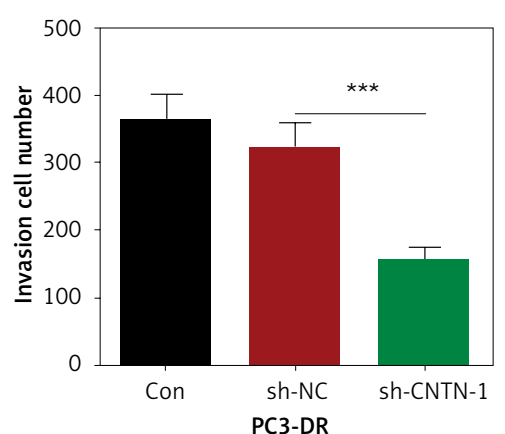

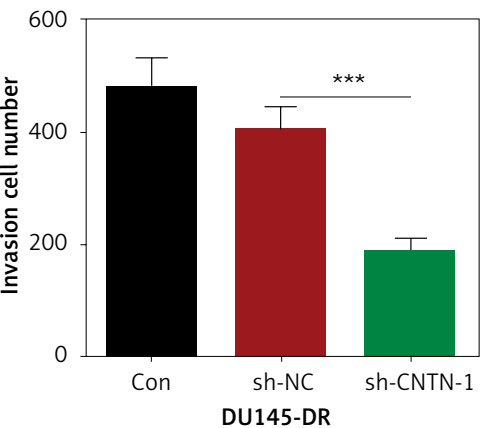

$E$

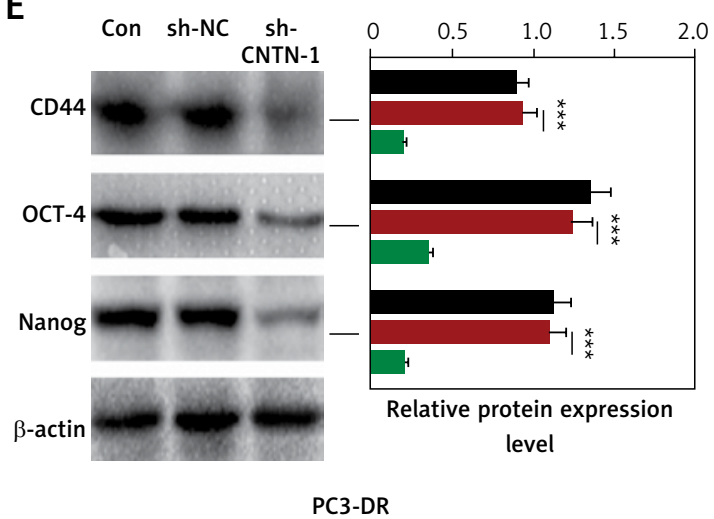

$\mathbf{F}$

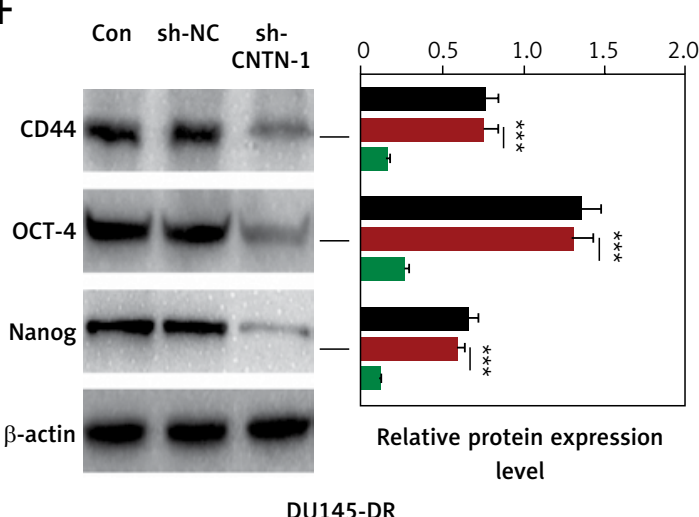

DU145-DR

Figure 4. Cont. C, D - Cell invasion was assessed by transwell assay. E, F - Protein levels of CD44, OCT-4 and Nanog were detected by western blotting

Data are presented as the mean $\pm S D .{ }^{*} P<0.05,{ }^{* *} p<0.01,{ }^{* *} p<0.001$. 


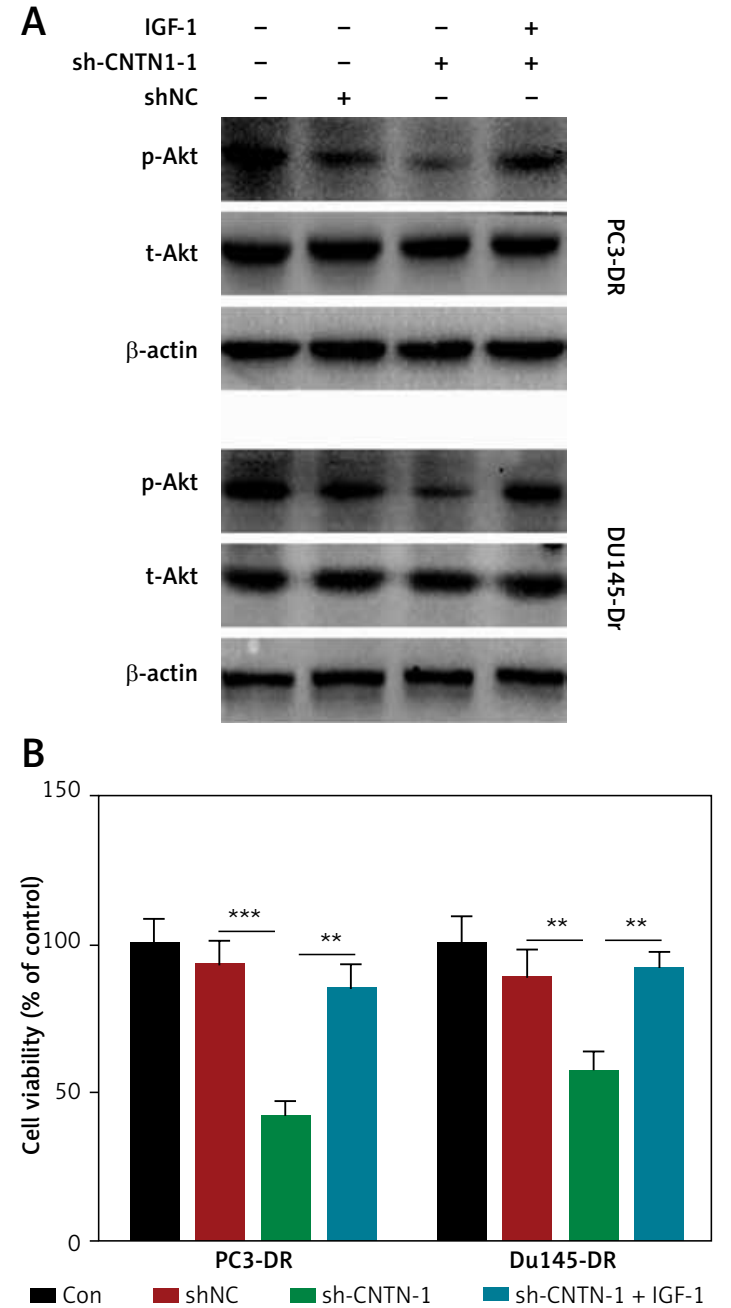

a variety of tumors [36]. To discover the potential mechanism of CNTN-1 regulating EMT, Dox resistance and malignant biological behaviors in $\mathrm{PCa}$, the PI3K/Akt signaling pathway was examined in this study. Western blotting analysis revealed that knockdown of CNTN-1 significantly downregulated p-Akt, indicating that the PI3K/Akt pathway was inhibited (Figure $5 \mathrm{~A}$ ). Furthermore, the PI3K activator IGF-1 evidently abrogated the inhibition of PI3K signaling by shCNTN-1 (Figure $5 \mathrm{~A}$ ), and abolished the effect of shCNTN-1 on PC3-DR and DU145-DR cells to docetaxel (Figure 5 B). Given these results, shCNTN-1 might reverse the EMT process which leads to malignant progression of PC3-DR and DU145-DR through blocking the PI3K/ Akt signaling.

\section{Silencing CNTN-1 increased the sensitivity} of Dox-resistant prostate cancer cells in vivo

To explore the effect of CNTN-1 on Dox-resistant PCa cells in vivo, a xenograft mouse model of PC3-DR cells which were stably transfected with shCNTN-1 or shNC was established. Seven days later, mice were given $10 \mathrm{mg} / \mathrm{kg}$ docetaxel intra-

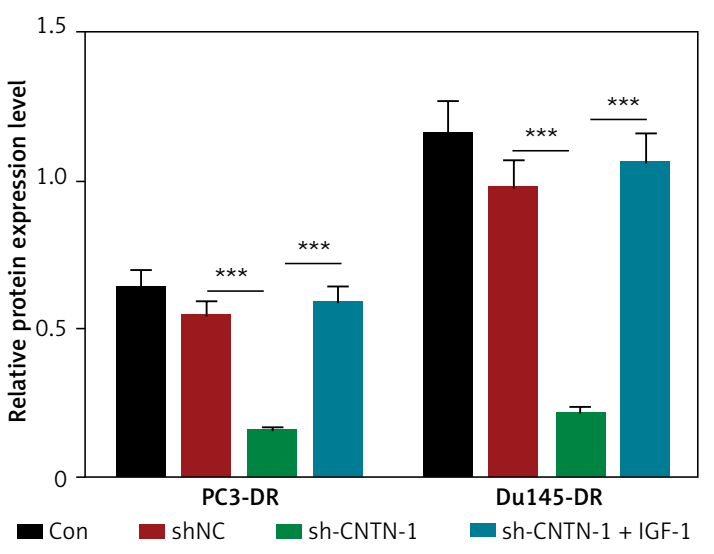

Figure 5. CNTN-1 regulates EMT and Dox resistance via mediating PI3K/Akt signaling. A - Protein levels of $p$-Akt and t-Akt were detected by western blotting. B - Cell viability was examined by CCK-8 assay

Data are presented as the mean $\pm S D .{ }^{*} P<0.05,{ }^{* *} p<$ $0.01,{ }^{* * *} p<0.001$.

peritoneally every 3 days. As shown in Figures $6 \mathrm{~A}$, $\mathrm{B}$, shCNTN-1 dramatically increased the inhibitory effect on docetaxel-induced tumor growth as evidenced by the reduction in tumor volume and final weight (Figure $6 \mathrm{C}$ ). As noted above, these data proved that shCNTN-1 sensitized Dox-resistant $\mathrm{PCa}$ cells in vivo. Moreover, coinciding with in vitro results, the knockdown of CNTN-1 significantly inhibited PI3K signaling, Akt phosphorylation and the expression level of interstitial markers (fibronectin, N-cadherin, vimentin), and elevated the expression level of the epithelial marker (E-cadherin) in comparison with the shNC group (Figure $6 \mathrm{D}$ ).

\section{Discussion}

Docetaxel is a microtubule polymerization-stabilizing agent commonly regarded as a first-line drug for chemotherapy in various malignant tumors including PCa [7-10]. However, drug resistance to docetaxel is a hindrance for $\mathrm{PCa}$ treatment. Further elucidation of the underlying mechanisms of chemoresistance associated with gene dysregulation can help to improve the un- 
A

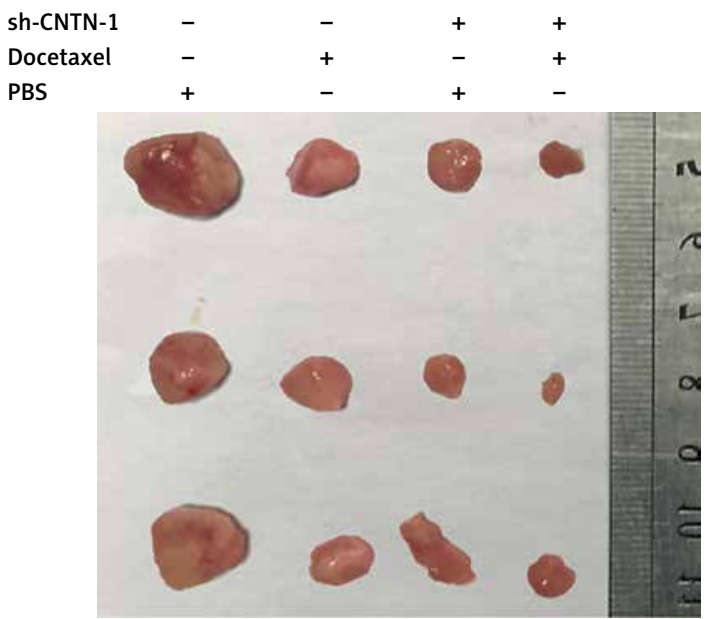

B

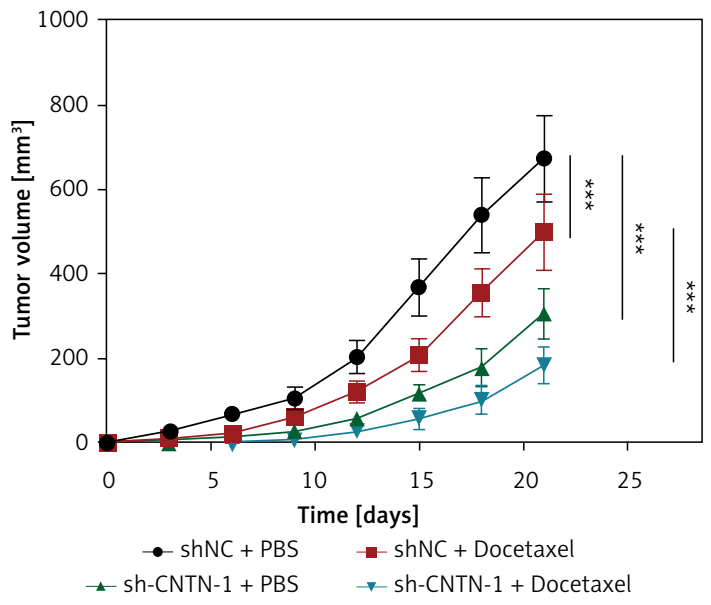

C

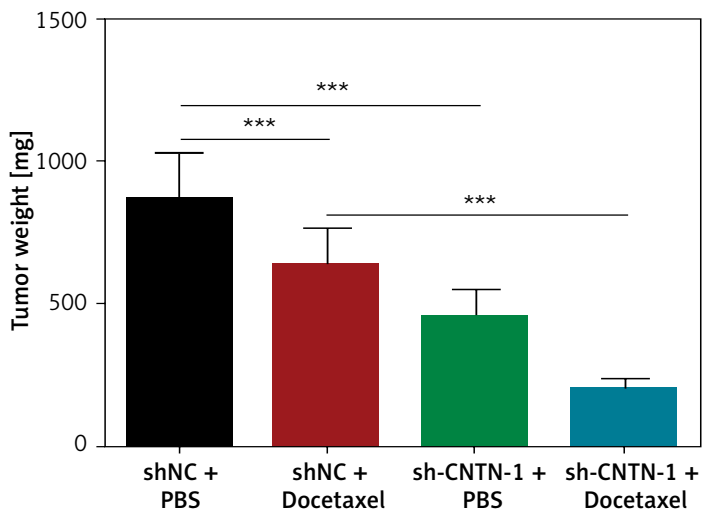

D

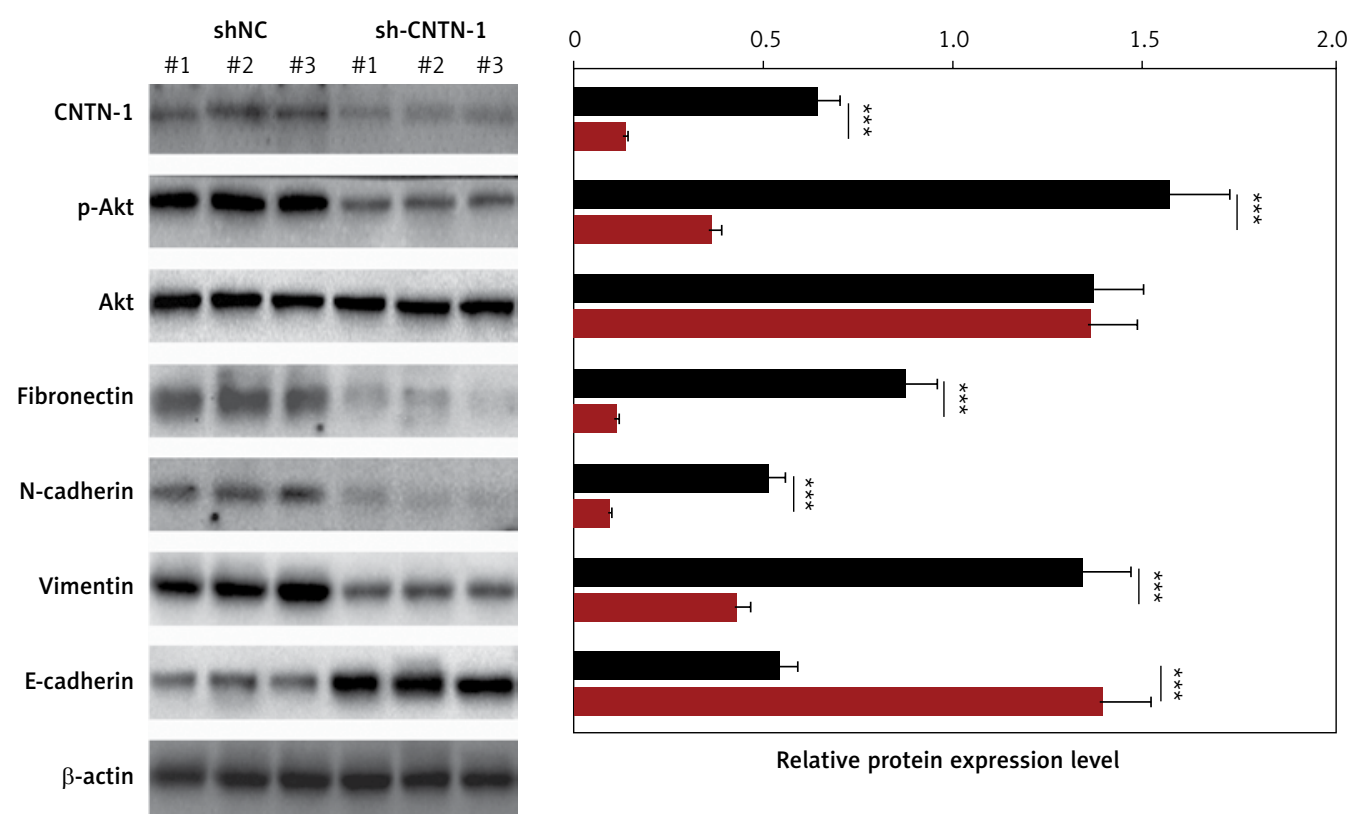

Figure 6. Silencing CNTN-1 increased the sensitivity of Dox-resistant prostate cancer cells in vivo. A - Images of tumor burden in xenograft mice in vivo assay using PC3-DR cells. B - Tumor volume in mice was monitored during the incubation time. C - Tumor weight was measured after mice were sacrificed. D - Protein levels of CNTN-1, p-Akt, t-Akt, fibronectin, vimentin, $\mathrm{N}$-cadherin and E-cadherin in PC3-DR xenograft tumors were detected by Western blotting

Data are presented as the mean $\pm S D .{ }^{*} P<0.05,{ }^{* *} p<0.01,{ }^{* * *} p<0.001$. 
derstanding of chemoresistance in multiple malignancies including prostate. To this end, we studied the function of CNTN-1 in Dox resistance in PCa and its potential mechanism in this research. Our results showed that CNTN-1 was upregulated in Dox-resistant PCa cells and silencing of CNTN-1 could reverse the resistance to Dox and inhibit the malignant process partially through impeding EMT/PI3K/Akt signaling.

CNTN-1, a member of the contactin subgroup of the immunoglobulin (Ig) superfamily, is a glycosylphosphatidylinositol (GPI)-anchored neuronal membrane protein abundant in the human brain and neural tissues that functions as a neuronal cell adhesion molecule [26]. Recently, CNTN-1 was demonstrated to promote the development of various cancers including lung, squamous, hepatocellular, gastric and prostate cancer [30, 37-41]. Aberrant CNTN-1 was discovered to aggravate tumor invasion, metastasis, EMT and chemoresistance in lung cancer [29]. In PCa, CNTN-1 exacerbated in vitro cell invasion and in vivo tumor growth and lung metastasis in PCa [30]. However, whether CNTN-1 can regulate docetaxel chemoresistance and the mechanism in PCa remains unclear.

EMT is crucial for chemoresistance development as well as migration and invasion in many malignancies [11-13, 15-18]. In our study, we demonstrated that knockdown of CNTN-1 inhibited the EMT phenotype with decreased EMT-related markers, and subsequently suppressed cell migration, invasion and expression of pluripotent markers in Dox-resistant PCa cells. Collectively, our data demonstrated that docetaxel-induced EMT was responsible for aggravated malignant tumor behaviors in $\mathrm{PCa}$, and was a possibly critical element in Dox resistance regulated by CNTN-1.

The PI3K/Akt signaling pathway is proved to be a promising target in cancer therapy for its contributions to cell proliferation, EMT and chemoresistance in various tumors. CNTN-1 promotes phosphorylation of Akt protein, thus increasing its activity, and an interrelation between CNTN-1 and the Akt signal has been reported $[28,29]$. In the present study, by silencing CNTN-1 in PCa, PI3K/ Akt signaling, EMT and Dox resistance were distinctly inhibited, and the effect of CNTN-1 knockdown was partially abolished by using the PI3K activator IGF-1 at the same time. These data suggest that CNTN-1 relies on PI3K/Akt signaling to mediate EMT and Dox resistance. Similarly, in vivo experiments showed that CNTN-1 silencing significantly increased docetaxel-induced inhibition of tumor growth and EMT by impeding PI3K/Akt signaling. However, there are still some limitations to our study. Detailed mechanisms of the CNTN-1/ $\mathrm{PI} 3 \mathrm{~K} / \mathrm{Akt}$ axis regulating chemoresistance of $\mathrm{PCa}$ cells are still elusive. However, all positive results remain experimental, and docetaxel resistance reversal of human prostate cancer is not found in clinical practice. CNTN-1 as a novel biomarker and target for overcoming Dox resistance remains to be further investigated in clinical studies. The mechanism of docetaxel resistance of human prostate cancer still requires in-depth study.

Although it remains essentially unknown what cellular mechanisms lead to CNTN-1 expression in $\mathrm{PCa}$, it is certain that these alterations also affect the expression of other important tumorigenic genes. Research into this mechanism will not only advance our understanding of tumorigenesis, but also enable development of better means of cancer diagnosis and therapy.

In conclusion, our findings indicated that CNTN-1 silencing inhibited the malignant biological behaviors including migration, invasion, and chemoresistance in Dox-resistant PCa cells via regulating the EMT process through blocking $\mathrm{PI} 3 \mathrm{~K} /$ Akt signaling, which provides new evidence that CNTN-1 could serve as a novel therapeutic target for overcoming Dox resistance in PCa treatment.

\section{Conflict of interest}

The authors declare no conflict of interest.

\section{References}

1. Siegel RL, Miller KD, Jemal A. Cancer statistics, 2015. CA Cancer J Clin 2015; 65: 5-29.

2. Cookson MS, Roth BJ, Dahm P, et al. Castration-resistant prostate cancer: AUA Guideline. J Urol 2015; 193: 491-9.

3. Saad F, Hotte SJ. Guidelines for the management of castrate-resistant prostate cancer. Can Urol Assoc J 2010; 4: 380-4.

4. Prajsner A, Chudek J, Szybalska A, et al. Socioeconomic determinants of prostate-specific antigen testing and estimation of the prevalence of undiagnosed prostate cancer in an elderly Polish population based on the PolSenior study. Arch Med Sci 2016; 12: 1028-35.

5. Francini E, Sweeney CJ. Docetaxel activity in the era of life-prolonging hormonal therapies for metastatic castration-resistant prostate cancer. Eur Urol 2016; 70: 410-2.

6. Walsh PC. Docetaxel and estramustine compared with mitoxantrone and prednisone for advanced refractory prostate cancer. N Engl J Med 2004; 173: 1513-20.

7. Brasso K, Thomsen FB, Schrader AJ, et al. Enzalutamide antitumour activity against metastatic castration-resistant prostate cancer previously treated with docetaxel and abiraterone: a multicentre analysis. Eur Urol 2015; 68: 317-24

8. Di LG, Bracarda S, Buonerba C, Aieta M, Mirone V. Poor survival in prostate cancer patients with primary refractoriness to docetaxel. Eur Urol 2014; 65: 505-7.

9. Galsky MD, Vogelzang NJ. Docetaxel-based combination therapy for castration-resistant prostate cancer. Ann Oncol 2010; 21: 2135-44.

10. Seruga B, Ocana A, Tannock IF. Drug resistance in metastatic castration-resistant prostate cancer. Nature Rev Clin Oncol 2011; 8: 12-23. 
11. Acloque H, Adams MS, Fishwick K, et al. Epithelial-mes enchymal transitions: the importance of changing cell state in development and disease. J Clin Invest 2009; 119: 1438-49.

12. Thiery JP, Acloque H, Huang RYJ, Nieto MA. Epithelial-mesenchymal transitions in development and disease. Cell 2009; 139: 871-90.

13. Kim MA, Lee HS, Lee HE, Kim JH, Yang HK, Kim WH. Prognostic importance of epithelial-mesenchymal transition-related protein expression in gastric carcinoma. Histopathology 2010; 54: 442-51.

14. Gawlik-Rzemieniewska N, Galilejczyk A, Krawczyk M, Bednarek I. Silencing expression of the NANOG gene and changes in migration and metastasis of urinary bladder cancer cells. Arch Med Sci 2016; 12: 889-97.

15. Tiwari N, Gheldof A, Tatari M, Christofori G. EMT as the ultimate survival mechanism of cancer cells. Semin Cancer Biol 2012; 22: 194-207.

16. Shang Y, Cai X, Fan D. Roles of epithelial-mesenchymal transition in cancer drug resistance. Curr Cancer Drug Targets 2013; 13: 963-72.

17. Voulgari A, Pintzas A. Epithelial-mesenchymal transition in cancer metastasis: mechanisms, markers and strategies to overcome drug resistance in the clinic. Biochim Biophys Acta 2009; 1796: 75-90.

18. Fischer KR, Durrans A, Lee S, et al. Epithelial-to-mesenchymal transition is not required for lung metastasis but contributes to chemoresistance. Nature 2015; 527: 472-6.

19. Witta SE, Gemmill RM, Hirsch FR, et al. Restoring E-cadherin expression increases sensitivity to epidermal growth factor receptor inhibitors in lung cancer cell lines. Cancer Res 2006; 66: 944-50.

20. Marín-Aguilera M, Codony-Servat J, Reig O, et al. Epi thelial-to-mesenchymal transition mediates docetaxel resistance and high risk of relapse in prostate cancer Mol Cancer Ther 2014; 13: 1270-84.

21. Zhou Z, Zhang L, Xie B, et al. FOXC2 promotes chemo resistance in nasopharyngeal carcinomas via induction of epithelial mesenchymal transition. Cancer Lett 2015 363: 137-45.

22. Zhang PF, Li KS, Shen Y, et al. Galectin-1 induces hepatocellular carcinoma EMT and sorafenib resistance by activating FAK/PI3K/AKT signaling. Cell Death Dis 2016; 7: e2201.

23. Bugide S, Gonugunta VK, Penugurti V, et al. HPIP promotes epithelial-mesenchymal transition and cisplatin resistance in ovarian cancer cells through PI3K/AKT pathway activation. Cell Oncol 2017; 40: 133-44.

24. Hanrahan K, O'Neill A, Prencipe M, et al. The role of epithelial-mesenchymal transition drivers ZEB1 and ZEB2 in mediating docetaxel-resistant prostate cancer. Mol Oncol 2017; 11: 251-65.

25. Puhr M, Hoefer J, Schäfer G, et al. Epithelial-to-mesenchymal transition leads to docetaxel resistance in prostate cancer and is mediated by reduced expression of miR200c and miR-205. Am J Pathol 2012; 181: 2188-201.

26. Falk J, Bonnon C, Girault JA, Faivre-Sarrailh C. F3/contactin, a neuronal cell adhesion molecule implicated in axogenesis and myelination. Biol Cell 2012; 94: 327-34.

27. Chen DH, Yu JW, Wu JG, Wang SL, Jiang BJ. Significances of contactin-1 expression in human gastric cancer and knockdown of contactin-1 expression inhibits invasion and metastasis of MKN45 gastric cancer cells. J Cancer Res Clin Oncol 2015; 141: 2109-20.
28. Yan J, Wong N, Hung C, Chen WX, Tang D. Contactin-1 reduces E-cadherin expression via activating AKT in lung cancer. PLoS One 2013; 8: e65463.

29. Zhang R, Sun S, Ji F, et al. CNTN-1 enhances chemoresistance in human lung adenocarcinoma through induction of epithelial-mesenchymal transition by targeting the PI3K/Akt pathway. Cell Physiol Biochem 2017; 43: 465-80.

30. Yan J, Ojo D, Kapoor A, et al. Neural cell adhesion protein CNTN1 promotes the metastatic progression of prostate cancer. Cancer Research 2016; 76: 1603.

31. Vinarskaja A, Yamanaka $M$, Ingenwerth $M$, Schulz WA. DNA methylation and the HOXC6 paradox in prostate cancer. Cancers 2011; 3: 3714-25.

32. O'Neill AJ, Maria P, Catherine D, et al. Characterisation and manipulation of docetaxel resistant prostate cancer cell lines. Mol Cancer 2011; 10: 126-6.

33. Polyak K, Weinberg RA. Transitions between epithelial and mesenchymal states: acquisition of malignant and stem cell traits. Nat Rev Cancer 2009; 9: 265-73.

34. Tam WL, Weinberg RA. The epigenetics of epithelial-mesenchymal plasticity in cancer. Nat Med 2013; 19: 1438-49.

35. Ye X, Weinberg RA. Epithelial-mesenchymal plasticity: a central regulator of cancer progression. Trends Cell Biol 2015; 25: 675-86.

36. Chiappini PB, de Medeiros IU, Lima LG, et al. Prognostic implications of phosphatidylinositol 3-kinase/AKT signaling pathway activation in gastric carcinomas. Arch Med Sci 2017; 13: 1262-8.

37. Su JL, Yang CY, Shih JY, et al. Knockdown of contactin-1 expression suppresses invasion and metastasis of lung adenocarcinoma. Cancer Res 2006; 66: 2553-61.

38. Liu P, Chen S, Wu W, et al. Contactin-1 (CNTN-1) overexpression is correlated with advanced clinical stage and lymph node metastasis in oesophageal squamous cell carcinomas. Japan J Clin Oncol 2012; 42: 612-8.

39. Qin XJ, Dai DJ, Gao ZG, Huan JL, Zhu L. Effect of lentivirus-mediated ShRNA targeting VEGFR-3 on proliferation, apoptosis and invasion of gastric cancer cells. Int J Mol Med 2011; 28: 761-8.

40. He-Ming WU, Cao W, Dongxia YE, Ren GX, Yu-Nong WU, Guo W. Contactin 1 (CNTN1) expression associates with regional lymph node metastasis and is a novel predictor of prognosis in patients with oral squamous cell carcinoma. Mol Med Rep 2012; 6: 265-70.

41. Li GY, Huang M, Pan TT, Jia WD. Expression and prognostic significance of contactin 1 in human hepatocellular carcinoma. Oncotargets Ther 2016; 9: 387-94. 